Muro de la Investigación, 2020(1), enero-junio

ISSN: 2523-2886

Doi: https://doi.org/10.17162/rmi.v5i1.1302

\title{
Efectividad del consumo de la hoja de guayaba sobre los niveles de glucosa en ratas diabéticas tipo 2
}

\author{
Effectiveness of the consumption of guava leaf on glucose levels in rats \\ with diabetes type 2
}

\author{
Betty Esther Díaz Correa ${ }^{1 a}$, Marco Antonio Chinchay Quito² \\ Universidad Peruana Unión, Lima, Perú ${ }^{12}$
}

Recibido: 14 de noviembre de 2019

Aceptado: 04 de enero de 2020

\section{Resumen}

Este trabajo de investigación tiene como objetivo evaluar la efectividad de la hoja de guayaba en polvo sobre los niveles de glucemia en ratas (Sprague - Dawley) inducidas a diabetes mellitus tipo 2 (DM2) con el fármaco estreptozotocina (STZ). Esta investigación tiene un enfoque cuantitativo, de diseño experimental, explicativo y de corte longitudinal. La muestra estuvo conformada por 40 ratas macho de la cepa Sprague -Dawley de 5 semanas, distribuidos en 3 grupos de tratamiento: grupo control no diabético, grupo experimental 2 y grupo experimental 3 respectivamente. Para el proceso de inducción de diabetes tipo 2, las ratas recibieron una cantidad de fructuosa al $10 \%$ por dos semanas, seguidamente se les administró el fármaco estreptozotocina (STZ) por vía intraperitoneal $(50 \mathrm{mg} / \mathrm{kg}$ ). Los grupos experimentales fueron alimentados por una sonda orogástrica, con una dosis de $500 \mathrm{mg} / \mathrm{Kg}$ y $1000 \mathrm{mg} / \mathrm{Kg}$ de hoja de guayaba en polvo, para los grupos experimentales que comprende el grupo 2 y el grupo 3, respectivamente, por un periodo de 3 semanas. Al finalizar el tratamiento, los grupos experimentales mostraron una disminución significativa de glicemia y un descenso de peso corporal. Este estudio concluye que la dosis de $1000 \mathrm{mg} / \mathrm{Kg}$ de la hoja de guayaba en polvo, presenta mayor disminución de glicemia, luego de su consumo.

Palabras clave: Diabetes mellitus tipo II, hoja de guayaba, glucosa, estreptozotocina.

\begin{abstract}
This research work aims to evaluate the evaluation of guava leaf powder on blood glucose levels in rats (Sprague-Dawley) induced by diabetes mellitus type 2 (DM2) with the drug streptozotocin (STZ). This research has a quantitative approach, experimental design, explanatory and longitudinal cut. The sample consisted of 40 male rats of the SpragueDawley strain of 5 weeks, distributed in 3 treatment groups: non-diabetic control group, experimental group 2 and experimental group 3 respectively. For the induction process of diabetes type 2, the rats received an amount of $10 \%$ fructose for two weeks, then the drug streptozotocin (STZ) was administered intraperitoneally $(50 \mathrm{mg} / \mathrm{kg}$ ). The experimental
\end{abstract}

\footnotetext{
a CORRESPONDENCIA AL AUTOR

Email: bethydiaz@upeu.edu.pe
} 
groups were fed by an orogastric tube, with a dose of 500mg / $\mathrm{Kg}$ and 1000mg / Kg of guava leaf powder, for the experimental groups comprising group 2 and group 3, respectively, for a period of 3 weeks. At the end of the treatment, the experimental groups showed a significant decrease in glycemia and a decrease in body weight. This study concludes that the dose of $1000 \mathrm{mg} / \mathrm{Kg}$ of the guava leaf powder shows a greater decrease in glycemia, after consumption.

Keywords: Diabetes mellitus Type 2, guava leaf, glucose, streptozotocin.

\section{Introducción}

La diabetes mellitus (DM) se produce por dos causas principales: la deficiencia de secreción de insulina y la resistencia a las células beta del cuerpo; también se caracteriza principalmente por una concentración anormal elevada de glucosa en sangre (Ibarra et al., 2006). Las estadísticas mencionan que existen cerca de 382 millones de personas que comprenden entre los 40 años y 59 años que viven con diabetes mellitus; más de 316 millo nes de personas padecen de tolerancia anormal a la glucosa y se estima que para el 2035 la cifra alcance a 471 millones de personas (FID, 2013).

En el Perú, la prevalencia de diabetes mellitus en personas que sobrepasan los 20 años de edad para el año 2005 fue de 2,8\%. Asimismo, los estudios de factores de riesgo para enfermedades no transmisibles realizados por la Dirección General de Epidemiología encontraron una prevalencia de DM de 2,8 a 3,9\% en ciudades de la costa y sierra: Lima, Callao, Villa el Salvador, Trujillo, Huancayo (Ramos et al., 2014).

Por su parte, la Organización Mundial de Salud relaciona a la diabetes tipos 2 (DM2) con el crecimiento y envejecimiento de la población, el incremento de la obesidad, malos hábitos alimenticios y estilos de vida sedentaria. Todo ello, implica un problema enorme en cuanto a la salud pública (Ibarra et al, 2006).

La diabetes mellitus tipo 2 (DM2) se considera como una enfermedad crónica degenerativa que presenta una tasa de mortalidad elevada, afectando la calidad de vida de la población a nivel mundial. Esta enfermedad se encuentra dentro del grupo de dolencias que

pueden llegar a causar invalidez por las múltiples complicaciones que presenta en el organismo y por la elevada morbi-mortalidad durante los últimos años. Estas complicaciones pueden darse muy aparte del estatus social, cultural y económico en los diferentes países (Torres et al., 2018). 


\section{Materiales y métodos}

El presente estudio se realizó en el bioterio de la Escuela de Medicina Humana de la Universidad Peruana Unión, que se encuentra ubicada en la Carretera Central Km19.5 Ñaña, Lurigancho, por un periodo de 8 semanas.

Participantes

El estudio consistió en 40 ratas macho de raza Sprague Dowley, adquiridos del bioterio de la Universidad Cayetano Heredia. Estas ratas, a partir del primer mes de edad, tuvieron un peso aproximado de 160gr-220gr, las cuales fueron inducidas a DM2 con estreptozotocina. Estas ratas fueron distribuidas de la siguiente manera.

Grupo 1, ratas sin diabetes mellitus tipo II.

Grupo 2, ratas con diabetes mellitus tipo II $+0.5 \mathrm{gr}$ de hoja de guayaba en polvo.

Grupo 3, ratas con diabetes mellitus tipo II + 1gr de hoja de guayaba en polvo.

Reactivos y/o métodos experimentales

Se empleó fructosa al $10 \%$ durante 2 semanas previas a la inducción de DM2, y $50 \mathrm{mg} / \mathrm{kg}$ del fármaco de la estreptozotocina (STZ) para que puedan contraer DM2.

Análisis de datos

En esta investigación se recolectaron los datos y se procesaron en Excel y en el Programa estadístico SPSS 24 para los análisis respectivos. En el estudio se realizó los siguientes análisis:

- Comparación de pesos a lo largo del tiempo de cada grupo de experimentación.

- Comparación del peso al final del tratamiento entre los grupos de experimentación

- Comparación de pesos entre el final y el inicio del tratamiento en cada grupo experimental.

- Comparación de niveles plasmáticos de glucosa a lo largo del tiempo de cada grupo de experimentación.

- Comparación de los niveles de glucosa plasmática al final del tratamiento entre los grupos de experimentación

- Comparación de niveles plasmáticos de glucosa entre el final y el inicio del tratamiento en cada grupo experimental. 
A fin de comparar las mediciones de cada grupo, se aplicó la prueba T de Student para los datos relacionados.

\section{Resultados}

\section{Tabla 1}

Valoración en el tiempo del peso corporal (g) según grupos de tratamiento

\begin{tabular}{|c|c|c|c|c|c|}
\hline \multirow[t]{2}{*}{ Grupos } & \multicolumn{5}{|c|}{ PESO CORPORAL (gr) } \\
\hline & Sem 1 & Sem 2 & Sem 4 & Sem 5 & Sem 6 \\
\hline $\begin{array}{c}\text { Grupo } \\
\text { control Nº1 }\end{array}$ & $\begin{array}{l}187.1 \pm \\
11.7\end{array}$ & $\begin{array}{l}196.9 \pm \\
15.4\end{array}$ & $\begin{array}{l}201.9 \pm \\
16.0\end{array}$ & $\begin{array}{l}212.9 \pm \\
12.5\end{array}$ & $229.8 \pm 11.8^{*}$ \\
\hline $\begin{array}{l}\quad \text { Grupo } \\
\text { experimental } \\
\mathrm{N}^{0} 2\end{array}$ & $\begin{array}{l}190.3 \pm \\
13.9\end{array}$ & $\begin{array}{l}201.1 \pm \\
14.4\end{array}$ & $7.6^{222.3 \pm}$ & 7.6 & $212.0 \pm 9.4^{* *}$ \\
\hline $\begin{array}{l}\quad \text { Grupo } \\
\text { experimental } \\
\mathrm{N}^{0} 3\end{array}$ & $\begin{array}{l}186.4 \pm \\
11.0\end{array}$ & $\begin{array}{l}198.5 \pm \\
11.5\end{array}$ & $\begin{array}{r}196.0 \pm \\
13.4^{* * *}\end{array}$ & $\begin{array}{l}164.5 \pm \\
11.2^{2}\end{array}$ & $165.0 \pm 7.1$ \\
\hline
\end{tabular}

La tabla 1 muestra la variación del peso corporal de los tres grupos de trabajo comparado con el peso de la semana anterior de cada grupo. Se puede observar que en las distintas semanas el peso corporal varía significativamente en el grupo 1 y los grupos experimentales 2 y 3 respectivamente. En el grupo control (1), se observa un incremento de peso corporal a lo largo de las semanas, mientras que en los grupos experimentales (grupos 2 y 3), la variación se da en disminución al peso corporal siendo el grupo 3 el que tiene una disminución más incrementada.

\section{Tabla 2}

Peso corporal final (g) y cambio del peso corporal durante el periodo de tratamiento 


\begin{tabular}{clc}
\hline Grupos & \multicolumn{1}{c}{ Peso final $(\mathbf{g})$} & $\begin{array}{c}\text { Cambio en el peso } \\
\text { corporal }(\mathbf{g})\end{array}$ \\
\hline Grupo control $\mathbf{N}^{\mathbf{0}} \mathbf{1}$ & $229.8 \pm 11.8$ & $27.9 \pm 7.5$ \\
Grupo experimental $\mathbf{N}^{\mathbf{0}} \mathbf{2}$ & $212.0 \pm 9.4$ & $-10.3 \pm 6.2^{a}$ \\
Grupo experimental $\mathbf{N}^{\mathbf{0} 3}$ & $165.0 \pm 7.1^{b, c}$ & $-31.0 \pm 8.5^{b, c}$ \\
\hline
\end{tabular}

${ }^{a} p<0.05$ comparado con Grupo 1

${ }^{b} p<0.05$ comparado con Grupo 2

${ }^{c} p<0.05$ comparado con Grupo 1

Se realizó la comparación de los pesos finales del grupo control y los dos grupos experimentales (tabla 4), encontrándose diferencia significativa entre los grupos $1-3,2-3$, los grupos 1 y 2 no mostraron diferencia significativa en sus pesos a la última semana de trabajo. Por otro lado, se calculó el cambio de peso corporal durante la fase de tratamiento (semanas 4 -6) encontrándose disminución de peso en los grupos de tratamiento (grupos 2 y 3) los cuales mostraron diferencia significativa entre sí y con el grupo control (grupo 1).

\section{Tabla 3}

Valoración de los niveles de glucosa en sangre (mg/dl) en los distintos grupos de experimentación

Grupos

Grupo control

No1

Grupo experimental $\mathbf{N}^{\mathbf{2} 2} \quad 11.8$

Grupo experimental $\mathbf{N}^{\mathbf{0} 3} \quad 17.0$

\section{Glicemia (mg/dl)}

\begin{tabular}{rlccc}
\hline Sem 1 & Sem 2 & Sem 4 & Sem 5 & Sem 6 \\
\hline $77.0 \pm$ & $86.9 \pm 5.6$ & $87.9 \pm$ & $98.8 \pm$ & $80.6 \pm$ \\
15.2 & & 12.7 & 31.3 & 7.3
\end{tabular}

$85.8 \pm \quad 93.0 \pm$ 10.9

$$
100.5 \pm
$$
$15.3 \&$

$\begin{array}{ccc}188.5 \pm & 142.3 \pm & 136.8 \pm \\ 26.2^{*} & 23.0^{* *} & 24.0 * * *\end{array}$

\begin{tabular}{llc}
$484.6 \pm$ & $240.5 \pm$ & $185.0 \pm$ \\
$93.2 \$$ & $73.4 \#$ & 28.3 \\
\hline
\end{tabular}

${ }^{*} \mathrm{p}<0.05$ comparado con Semana 2

** $\mathrm{p}<0.05$ comparado con Semana 4

$* * * \mathrm{p}<0.05$ comparado con Semana 5

${ }^{\&} \mathrm{p}<0.05$ comparado con Semana 1

$\$_{\mathrm{p}}<0.05$ comparado con Semana 2 


\section{${ }^{\#} \mathrm{p}<0.05$ comparado con Semana 4}

La tabla 3 muestra los niveles de glucosa a lo largo de cada semana en cada grupo experimental. Se analizaron las variaciones de la glicemia, semana a semana, comparando cada valor con la semana anterior dentro del mismo grupo. Se observa que tras la inducción con STZ, los individuos de los grupos experimentales aumentaron drásticamente su concentración plasmática de glucosa (semana 4) con valores superiores a 130mg/dl lo que nos indica que se tornaron diabéticas. Una vez iniciado el tratamiento con la preparación de hojas de guayaba en polvo en las respectivas dosis, se verificó una disminución significativa de la glicemia (grupos 2 y 3). El grupo control no tuvo variaciones significativas en los valores de glicemia a lo largo de las semanas de experimentación.

Tabla 4

Valores de glicemia final $(\mathrm{mg} / \mathrm{dl})$ y cambio de la glicemia durante el periodo de tratamiento

Grupos

Grupo control $\mathbf{N}^{\mathbf{0}} \mathbf{1}$

Grupo experimental No2

Grupo experimental No3
Glicemia final (mg/dL)

\section{Cambio en la glicemia} (mg/dL)
$-7.3 \pm 3.5$
$-51.7 \pm 7.5^{a}$
$80.6 \pm 7.3$
$136.8 \pm 24.0^{\mathrm{a}}$

$185.0 \pm 28.3^{\mathrm{b}, \mathrm{c}}$

$-299.6 \pm 20.4^{\mathrm{b}, \mathrm{c}}$

${ }^{\mathrm{a}} \mathrm{p}<0.05$ comparado con Grupo 1
${ }^{b} \mathrm{p}<0.05$ comparado con Grupo 2
${ }^{c} \mathrm{p}<0.05$ comparado con Grupo 1

Se realizó la comparación de los valores de glicemia finales entre los tres grupos de trabajo encontrándose diferencias significativas entre los dos grupos de experimentación y el grupo control. Adicionalmente, se calculó el cambio de la glicemia durante la fase de tratamiento con las hojas de guayaba, verificándose que el grupo 3 (dosis $1 \mathrm{~g}$ diario de hojas de guayaba) tuvo el mayor cambio significativo comparado al grupo 2 y al control. El grupo 2 (dosis 0.5 g diarios) tuvo una disminución moderada en los valores de glicemia, este cambio también fue significativo en relación a la variación dentro del grupo control. 


\section{Discusión}

Diabetes mellitus (DM) es un trastorno metabólico con múltiples síntomas caracterizados por hiperglucemia severa junto con el metabolismo alterado de hidratos de carbono, grasas y proteínas, el cual es el resultado de defectos en la secreción de insulina / acción de la insulina o ambos. Es considerada una de las epidemias de más rápido crecimie nto a nivel mundial (415 millones de pacientes).

En este trabajo los animales fueron inducidos con estreptozotocina para obtener diabetes mellitus tipo 2 (grupo 2 y grupo 3), estos grupos presentaron un descenso de peso corporal significativo $(\mathrm{p}<0.05)$, durante la fase de tratamiento con la hoja de guayaba en polvo (tabla 1); al igual que Mora (2009) y Dyson (2018), presentaron descensos de peso debido a la diabetes mellitus tipo 2; esto ocurre por la incapacidad del ingreso de glucosa en tejidos insulino-dependientes como los tejidos adiposos y muscular; este cuadro metabólico intensificaría vías metabólicas como la gluconeogénesis hepática, lipólisis en el tejido adiposo y proteólisis en el tejido muscular, lo cual conllevaría a una sustancial pérdida de masa del organismo (Mora y Aragón, 2009; Dyson, 2018).

Asimismo, los animales de los grupos experimentales llegaron a tener valores de glicemia hasta $450 \mathrm{mg} / \mathrm{dl}$ evidenciando un cuadro semejante a pacientes diabéticos no controlados o pobremente controlados. La administración de hoja de Psidium guajaba redujo notablemente los niveles de glucosa en sangre en un $27.6 \%$ y en un $61 \%$ en el grupo 2 y grupo 3 respectivamente, al finalizar el tratamiento (tabla 3). Resultados similares, a lo encontrado por-Jayachandran et al., 2018, donde la suplementación con extractos de hoja de guayaba en ratas diabéticas suprimía la acción generada por la estreptozotocina, inhibie ndo también el estado inflamatorio generado por este fármaco, permitiendo así la recuperación de las células beta pancreáticas, lo que conduciría a la disminución del $53 \%$ de la hiperglicemia al finalizar el tratamiento. Similar Vinayagam et al. (2018), demostraron que la administración oral de Psidium guajaba $(200 \mathrm{mg} / \mathrm{kg}$ ) aumentó considerablemente los niveles de insulina, glucógeno y hexocinasa reduciendo los niveles de glucosa en sangre en ratas diabéticas tipo 2 en un 50\% al finalizar el tratamiento; por otro lado, el estudio de Inocente et al. revela que, al finalizar el tratamiento, la concentración de glucosa en sangre disminuyó en un 80\%, asimismo, se evaluó la actividad antidiabética de los extractos acuoso 
y etanólico que fueron obtenidos a partir de las hojas de Psidium guajava L (Jayachandran et al., 2018; Vinayagam et al., 2018; Angel, 2015).

$\mathrm{Al}$ hacer el análisis físico-químico de la hoja de guayaba se encontró $17.7 \%$ de fibra bruta y $68.9 \%$ de carbohidratos totales, conteniendo probablemente entre los componentes de la fibra. De acuerdo a los estudios de Álvarez et al. (2006), Narenjkar et al. (2011) y Sánchez et al. (2004), señalan que la hoja de guayaba está compuesta por 3 tipos de flavonoides tales como la mirecetina, kaempferol y quercetina, este último flavonoide se encuentra en un $45 \%$ en la composición nutricional de la hoja de guayaba. Asimismo, tiene una actividad hipoglucemiante, es decir, que ayuda a inhibir la alfa-glucosidasa y reduce los niveles posprandiales de glucosa (Alvarez, 2006; Narenjkar et al., 2011; Blanco et al, 2004). Al ser un producto natural, es necesario realizar anális is adicionales sobre la hoja de guayaba de origen nativo los cuales puedan arrojar luz sobre la búsqueda de fitoquímicos eficaces en el tratamiento de la diabetes mellitus tipo 2.

En conclusión, el tratamiento con hoja de guayaba en polvo (Psidium guajava) no mantiene el peso de las ratas machos (Sprague Dawley) inducidas a diabetes mellitus tipo 2(DM2) con estreptozotocina. El tratamiento con hoja de guayaba en polvo (Psidium guajava) disminuye significativamente los niveles de glicemia de las ratas machos (Sprague Dawley) inducidas a diabetes mellitus tipo 2(DM2) con estreptozotocina.

\section{Referencias}

Alvarez, V., Hernández, S., Hernández, G., Víctor, A., Engleman, M., Garza, M. (2006). Cinética de acumulación y distribución de flavonoides en guayaba (Psidium guajava L.). Agrociencia, 40(1): 109-115.

Bartrina, J. (2018). Nutrición hospitalaria presentación, 34:0-1.

Blanco-Hernandez, Ruiz-Ramos, M., Sánchez-Rodriguez y Mendoza-Núñez, M. (2004). Lipoperóxidos, actividad antioxidante y factores pro-oxidantes en adultos mayores con diabetes mellitus tipo 2*. Bioquimia, 29(4):118-125.

Carreto, N., Fraga, M., Chon, O., López, S. (2005). Introducción a la fisiología pericárdica, 1-30.

Crespo Retes, I. (2008). Guía peruana de diagnóstico, control y tratamiento de la diabetes mellitus tipo 2. Sociedad Peruana de Endocrinología, 2-134. Recuperado de: 
http $/ /$ www.endocrinoperu.org/pdf/Guia Peruana de Diagnostico Control y Tratamiento de la Diabetes Mellitus 2008.pdf

Dirección General de Epidemiología. (2013). Situación de la vigilancia de diabetes en el Perú al I semestre de 2013. Bol Epidemiológico, 22(39):825-8.

Dyson, P., Twenefour, D., Breen, C., Duncan, A., Elvin, E., Goff, L. (2018). Evidencebased nutrition guidelines for the prevention and management of diabetes. Diabetes $U K, 1$.

Ertugrul, N., Hasegawa, R., Soong, W., Gayler, J., Kloeden, S., Kahourzade, S. (2015). A Novel Tapered Rotating Electrical Machine Topology Utilizing Cut Amorphous Magnetic Material. IEEE Transactions on magnetics, 51(7).

Federación Internacional de la Diabetes. (2015). Atlas de la diabetes de la FDI. International Diabetes Federation, 47-51.

FID. (2013). Atlas de Diabetes. Available from: http//www.fmdiabetes.org/fmd/des/SP_6E_Atlas_Full.pdf

Figueredo, E. (2016). Tratamiento actual de la diabetes mellitus tipo 2. Correo cientifico médico, 20(1):98-121.

Figueroa-Suarez, M., Cruz-Toledo, J., Ortiz-Aguirre, A., Lagunes-Espinoza, A., JimenezLuna, J. y Rodriguez-Moctezuma, J. (2014). Estilo de vida y control metabólico en diabéticos del programa DiabetIMSS. Gaceta médica de Mexico, 150:29-34.

Guadalupe, M., Martínez, C., Gutiérrez, S., Gabriela, M., Craviotto, L., Rosas, A. (2012). Manejo de la hiperglucemia en el paciente hospitalizado. Medicina interna de México, 28(2):124-53.

Ibarra R, Luisa M, González B, Margarita C, Meda G, Claudia B. (2006). Diabetes , estrés oxidativo y antioxidantes.

Inocente-Camones, M., Guija-Poma, E., Zarzosa-Norabuena, E., Loja-Herrera, B., PoncePardo, J. (2015). Efecto hipoglicemiante de los extractos acuoso y etanólico de Psidium guajava L. (Guayaba) en ratas diabéticas inducidas por aloxano. Horiz Med, 15(2):41-8.

Jayachandran, M., Vinayagam, R., Ambati, R., Xu, B., Chung, S. (2018). Guava leaf extract diminishes hyperglycemia and oxidative stress, prevents $\beta$-cell death, 
inhibits inflammation, and regulates NF-kB signaling pathway in STZ induced diabetic rats. Biomed Res Int.

Khan, H., Rajendran, D., Bai, M., Sorimuthu, S. (2013). Protective effect of Psidium guajava leaf extract on altered carbohydrate metabolism in streptozotocin-induced diabetic rats. J Diet Suppl, 10(4):335-44.

Mayorga, U., Fernando, C., Olga, N., Zegarra, T., Miriam, L., Zegarra, T. (2004). Complicaciones tardías en diabetes mellitus tipo 2 en el Hospital II Essalud Cañete. Revista médica herediana, 15(2):64-9.

Mediavilla-Bravo, J. (2016). ¿Qué estrategias de combinación de fármacos son recomendables en el tratamiento de personas con diabetes y mal control glucémico? Guía Actual en Diabetes Mellit tipo 2. ;68-70. Recuperado de: http//red gdps.org/gestor/upload/GUIA2016/Guia_Actualizacion_2016.pdf

Ministerio de Salud. (2010). Guia clinica Diabetes Mellitus tipo 2. Vol. 1.

Mora, A., Aragon, D., Ospina, L. (2009). Caracterización del estrés oxidativo en ratas wistar diabéticas por estreptozotocina. Vitae: Revista de la facultad de quimica farmaceutica, 16(3): 311-319.

Müller, U., Stübl, F., Schwarzinger, B., Sandner, G., Iken, M., Himmelsbach, M. (2018). In vitro and in vivo inhibition of intestinal glucose transport by guava (psidium guajava) extracts. Mol Nutr Food Res, 62(11):1-11.

Narenjkar, J., Roghani, M., Alambeygi, H., Sedaghati, F., Mellitus. (2011). The Effect of the Flavonoid Quercetin on Pain Sensation in Diabetic Rats. Basic and clinical neuroscience, 2(3):51-57.

Olmedo, J. (2017). Diabetes gestacional: conceptos actuales. Current concepts, 85(6):38090.

Oré, R. (2011). Disminucion del daño oxidativo y efecto hipoglicemiante de la maca ( Lepidium meyenii Walp ) en ratas con diabetes inducida por streptozotocina. $A n$ Fac Med., 72(1):7-11.

Oviedo Mota, M. (2004). Guía clínica para el diagnóstico y tratamiento de la diabetes mellitus tipo 2, 41:1-21.

Pacheco, M., Poma, E., Reyes, N. (209). Antioxidants properties of Guava (Psidium guajava L.). Rev Soc Quím Perú, 75(2):228-34. 
Ramos, W., López, T., Revilla, L., More, L., y Huamaní, M. (2012). Resultados de la vigilancia epidemiológica de diabetes mellitus en hospitales notificantes del Perú, 2012. Revista peruana de medicina experimental y salud pública, 31(1):9-15.

Ramos-Ibarra, M., Bautista-González, C.; Goméz-Meda, B.; y Zamora-Pérez, A. (2006). Diabetes, estrés oxidativo y antioxidantes. Revista de educación bioquímica, VIII(1):7-15.

Ruiz-Ramos, M., Escolar-Pujolar, A., Mayoral-Sánchez, E., Laureano, F., FernándezFernández, I. (2006). La diabetes mellitus en España: mortalidad, prevalencia, incidencia, costes económicos y desigualdades. Gaceta sanitaria, 20(Supl 1):15-24.

Salazar-García, M. (2010). Politécnico nacional, 93.

Torres, O., Bañuelos, D., Villaseñor, A., Ruiz, P., Hodgers-Félix, R. (2018). Medicina general y de familia: Carta al Editor, 5(3):128-9.

Trejo-González, N., Chirino-Galindo, G., Palomar-Morales, M. (2015). Capacidad antiteratogénica del resveratrol en diabetes inducida por estreptozotocina en ratas. Rev. Peru. Med. Exp. Salud Pública, 32(3):457-63.

Vélez, S., Gómez, L., Tapia, D., Guerrero, E., Morán J. Estandarización del modelo de diabetes experimental inducida por estreptozotocina en ratas Sprague-Dawley. Rev Médico Científica, 28(1):4-13.

Vinayagam, R., Jayachandran, M., Chung, S., Xu, B. (2018). Guava leaf inhibits hepatic gluconeogenesis and increases glycogen synthesis via AMPK/ACC signaling pathways in streptozotocin-induced diabetic rats. Biomedicine \& Pharmacotherapy, 103, 1012-7. Recuperado de: https://doi.org/10.1016/j.biopha.2018.04.127

White, E.G. (1971). Consejos para los maestros. Buenos Aires: Argentina: ACES.

Zapata, K., Cortes, F., Rojano, B. (2013). Polifenoles y actividad antioxidante del fruto de guayaba agria (Psidium araca). Inf Tecnol, 24(5):103-12. 